\title{
Secure Data Retrieval for Decentralized Disruption-Tolerent Military Networks
}

\author{
Savita S. Kulkumatagi
}

\begin{abstract}
Mobile nodes in military environments such as a battlefield or a hostile region are likely to suffer from intermittent network connectivity and frequent partitions. Disruption-tolerant network (DTN) technologies are becoming successful solutions that allow wireless devices carried by soldiers to communicate with each other and access the confidential information or command reliably by exploiting external storage nodes. Disruption- tolerant network (DTN) technologies are becoming successful solutions that allow nodes to communicate with each other in these extreme networking environments. Typically, when there is no end-toend connection between a source and a destination pair, the messages from the source node may need to wait in the intermediate nodes for a substantial amount of time until the connection would be eventually established. The concept of attribute-based encryption (ABE) is a promising approach that fulfills the requirements for secure data retrieval in DTNs. Especially, Ciphertext-Policy ABE (CP-ABE) provides a scalable way of encrypting data such that the encryptor defines the attribute set that the decryptor needs to possess in order to decrypt the ciphertext. Thus, different users are allowed to decrypt different pieces of data per the security policy.
\end{abstract}

Keywords--- Disruption-Tolerant Network (DTN), Attribute-Based Encryption (ABE), Ciphertext-Policy ABE $(C P-A B E)$.

\section{INTRODUCTION}

$I_{d}^{N}$ $\mathrm{N}$ many military network scenarios, connections of wireless devices carried by soldiers may be temporarily disconnected by jamming, environmental factors, and mobility, especially when they operate in hostile environments. Disruptiontolerant network (DTN) technologies are becoming successful solutions that allow nodes to communicate with each other in these extreme networking environments. Typically, when there is no end-to-end connection between a source and a destination pair, the messages from the source node may need to wait in the intermediate nodes for a substantial amount of time until the connection would be eventually established.

Roy and Chuah introduced storage nodes in DTNs where data is stored or replicated such that only authorized mobile nodes can access the necessary information quickly and efficiently. Many military applications require increased protection of confidential data including access control methods that are cryptographically enforced.

Savita S. Kulkumatagi, M.Tech Student, Department of Computer Science and Engineering, SDMCET, Dharwad, VTU, Karnataka, India. E-mai:savita.saran@yahoo.co.in DOI:10.9756/BIJSESC.8283
In many cases, it is desirable to provide differentiated access services such that data access policies are defined over user attributes or roles, which are managed by the key authorities. For example, in a disruption-tolerant military network, a commander may store a confidential information at a storage node, which should be accessed by members of "Battalion 1" who are participating in "Region 2." In this case, it is a reasonable assumption that multiple key authorities are likely to manage their own dynamic attributes for soldiers in their deployed regions or echelons, which could be frequently changed (e.g., the attribute representing current location of moving soldiers). We refer to this DTN architecture where multiple authorities issue and manage their own attribute keys independently as a decentralized DTN. The concept of attribute-based encryption (ABE) is a promising approach that fulfills the requirements for secure data retrieval in DTNs. ABE features a mechanism that enables an access control over encrypted data using access policies and ascribed attributes among private keys and ciphertexts. Especially, ciphertextpolicy $\mathrm{ABE}(\mathrm{CP}-\mathrm{ABE})$ provides a scalable way of encrypting data such that the encryptor defines the attribute set that the decryptor needs to possess in order to decrypt the ciphertext. Thus, different users are allowed to decrypt different pieces of data per the security policy. However, the problem of applying the ABE to DTNs introduces several security and privacy challenges. Since some users may change their associated attributes at some point (for example, moving their region), or some private keys might be compromised, key revocation (or update) for each attribute is necessary in order to make systems secure. However, this issue is even more difficult, especially in ABE systems, since each attribute is conceivably shared by multiple users (henceforth, we refer to such a collection of users as an attribute group). This implies that revocation of any attribute or any single user in an attribute group would affect the other users in the group. For example, if a user joins or leaves an attribute group, the associated attribute key should be changed and redistributed to all the other members in the same group for backward or forward secrecy. It may result in bottleneck during rekeying procedure, or security degradation due to the windows of vulnerability if the previous attribute key is not updated immediately. Another challenge is the key escrow problem. In CP-ABE, the key authority generates private keys of users by applying the authority's master secret keys to users' associated set of attributes. Thus, the key authority can decrypt every ciphertext addressed to specific users by generating their attribute keys. If the key authority is compromised by adversaries when deployed in the hostile environments, this could be a potential threat to the data confidentiality or privacy especially when the data is highly sensitive. The key escrow is an inherent problem even in the multiple-authority systems as long as each 
key authority has the whole privilege to generate their own attribute keys with their own master secrets. Since such a key generation mechanism based on the single master secret is the basic method for most of the asymmetric encryption systems such as the attribute- based or identity-based encryption protocols, removing escrow in single or multiple-authority $\mathrm{CP}-\mathrm{ABE}$ is a pivotal open problem. The last challenge is the coordination of attributes issued from different authorities. When multiple authorities manage and issue attribute keys to users independently with their own master secrets, it is very hard to define fine-grained access policies over attributes issued from different authorities

\section{MODULE DESCRIPTION}

\section{A. Key Authorities}

They are key generation centers that generate public/secret parameters for $\mathrm{CP}-\mathrm{ABE}$. The key authorities consist of a central authority and multiple local authorities. We assume that there are secure and reliable communication channels between a central authority and each local authority during the initial key setup and generation phase. Each local authority manages different attributes and issues corresponding attribute keys to users. They grant differential access rights to individual users based on the users' attributes. The key authorities are assumed to be honest-but-curious. That is, they will honestly execute the assigned tasks in the system, however they would like to learn information of encrypted contents as much as possible

\section{B. Storage Node}

This is an entity that stores data from senders and provide corresponding access to users. It may be mobile or static. Similar to the previous schemes, we also assume the storage node to be semi-trusted, that is honest-but-curious.

\section{Sender}

This is an entity who owns confidential messages or data (e.g., a commander) and wishes to store them into the external data storage node for ease of sharing or for reliable delivery to users in the extreme networking environments. A sender is responsible for defining (attribute based) access policy and enforcing it on its own data by encrypting the data under the policy before storing it to the storage node.

\section{CP-ABE Method}

In Ciphertext Policy Attribute based Encryption scheme, the encryptor can fix the policy, who can decrypt the encrypted message. The policy can be formed with the help of attributes. In $\mathrm{CP}-\mathrm{ABE}$, access policy is sent along with the ciphertext. We propose a method in which the access policy need not be sent along with the ciphertext, by which we are able to preserve the privacy of the encryptor. This techniques encrypted data can be kept confidential even if the storage server is untrusted; moreover, our methods are secure against collusion attacks. Previous Attribute- Based Encryption systems used attributes to describe the encrypted data and built policies into user's keys; while in our system attributes are used to describe a user's credentials, and a party encrypting data determines a policy for who can decrypt.

\section{SOFTWARE ENVIRONMENT}

\section{A. Features of. Net}

Microsoft .NET is a set of Microsoft software technologies for rapidly building and integrating XML Web services, Microsoft Windows-based applications, and Web solutions. The .NET Framework is a language-neutral platform for writing programs that can easily and securely interoperate. There's no language barrier with .NET: there are numerous languages available to the developer including Managed $\mathrm{C}++$, C\#, Visual Basic and Java Script. The .NET framework provides the foundation for components to interact seamlessly, whether locally or remotely on different platforms. It standardizes common data types and communications protocols so that components created in different languages can easily interoperate.

"NET" is also the collective name given to various software components built upon the .NET platform. These will be both products (Visual Studio.NET and Windows.NET Server, for instance) and services (like Passport, .NET My Services, and so on).

\section{B. Managed Code}

The code that targets .NET, and which contains certain extra Information - "metadata" - to describe itself. Whilst both managed and unmanaged code can run in the runtime, only managed code contains the information that allows the CLR to guarantee, for instance, safe execution and interoperability.

\section{Managed Data}

With Managed Code comes Managed Data. CLR provides memory allocation and Deal location facilities, and garbage collection. Some .NET languages use Managed Data by default, such as C\#, Visual Basic.NET and JScript.NET, whereas others, namely $\mathrm{C}++$, do not. Targeting CLR can, depending on the language you're using, impose certain constraints on the features available. As with managed and unmanaged code, one can have both managed and unmanaged data in .NET applications - data that doesn't get garbage collected but instead is looked after by unmanaged code.

\section{Common Type System}

The CLR uses something called the Common Type System (CTS) to strictly enforce type-safety. This ensures that all classes are compatible with each other, by describing types in a common way. CTS define how types work within the runtime, which enables types in one language to interoperate with types in another language, including cross-language exception handling. As well as ensuring that types are only used in appropriate ways, the runtime also ensures that code doesn't attempt to access memory that hasn't been allocated to it.

\section{E. Common Language Specification}

The CLR provides built-in support for language interoperability. To ensure that you can develop managed code that can be fully used by developers using any programming language, a set of language features and rules for using them called the Common Language Specification (CLS) has been defined. Components that follow these rules and expose only 
CLS features are considered CLS-compliant.

\section{F. The Class Library}

.NET provides a single-rooted hierarchy of classes, containing over 7000 types. The root of the namespace is called System; this contains basic types like Byte, Double, Boolean, and String, as well as Object. All objects derive from System. Object. As well as objects, there are value types. Value types can be allocated on the stack, which can provide useful flexibility. There are also efficient means of converting value types to object types if and when necessary.

The set of classes is pretty comprehensive, providing collections, file, screen, and network I/O, threading, and so on, as well as XML and database connectivity.

The class library is subdivided into a number of sets (or namespaces), each providing distinct areas of functionality, with dependencies between the namespaces kept to a minimum.

\section{G. Languages Supported By.Net}

The multi-language capability of the .NET Framework and Visual Studio .NET enables developers to use their existing programming skills to build all types of applications and XML Web services. The .NET framework supports new versions of Microsoft's old favorites Visual Basic and C++ (as VB.NET and Managed $\mathrm{C}++$ ), but there are also a number of new additions to the family.

Visual Basic .NET has been updated to include many new and improved language features that make it a powerful object-oriented programming language. These features include inheritance, interfaces, and overloading, among others. Visual Basic also now supports structured exception handling, custom attributes and also supports multi-threading.

Visual Basic .NET is also CLS compliant, which means that any CLS-compliant language can use the classes, objects, and components you create in Visual Basic .NET.

Managed Extensions for $\mathrm{C}++$ and attributed programming are just some of the enhancements made to the $\mathrm{C}++$ language. Managed Extensions simplify the task of migrating existing $\mathrm{C}++$ applications to the new .NET Framework.

$\mathrm{C} \#$ is Microsoft's new language. It's a C-style language that is essentially " $\mathrm{C}++$ for Rapid Application Development". Unlike other languages, its specification is just the grammar of the language. It has no standard library of its own, and instead has been designed with the intention of using the .NET libraries as its own.

Microsoft Visual J\# .NET provides the easiest transition for Java-language developers into the world of XML Web Services and dramatically improves the interoperability of Java-language programs with existing software written in a variety of other programming languages.

Active State has created Visual Perl and Visual Python, which enable .NET-aware applications to be built in either Perl or Python. Both products can be integrated into the Visual Studio .NET environment. Visual Perl includes support for Active State's Perl Dev Kit.
Other languages for which .NET compilers are available include
1) FORTRAN
2) $\mathrm{COBOL}$
3) Eiffel

\begin{tabular}{|r|c|}
\hline ASP.NET & \\
XML WEB & Windows Forms \\
SERVICES & \\
\hline \multicolumn{2}{|c|}{ Base Class Libraries } \\
\hline \multicolumn{2}{|c|}{ Common Language Runtime } \\
\hline \multicolumn{2}{|c|}{ Operating System } \\
\hline
\end{tabular}

Figure1: Net Framework

C\#.NET is also compliant with CLS (Common Language Specification) and supports structured exception handling. CLS is set of rules and constructs that are supported by the CLR (Common Language Runtime). CLR is the runtime environment provided by the .NET Framework; it manages the execution of the code and also makes the development process easier by providing services.

C\#.NET is a CLS-compliant language. Any objects, classes, or components that created in C\#.NET can be used in any other CLS-compliant language. In addition, we can use objects, classes, and components created in other CLScompliant languages in C\#.NET .The use of CLS ensures complete interoperability among applications, regardless of the languages used to create the application.

\section{H. Constructors And Destructors}

Constructors are used to initialize objects, whereas destructors are used to destroy them. In other words, destructors are used to release the resources allocated to the object. In C\#.NET the sub finalize procedure is available. The sub finalize procedure is used to complete the tasks that must be performed when an object is destroyed. The sub finalize procedure is called automatically when an object is destroyed. In addition, the sub finalize procedure can be called only from the class it belongs to or from derived classes.

\section{Garbage Collection}

Garbage Collection is another new feature in C\#.NET. The .NET Framework monitors allocated resources, such as objects and variables. In addition, the .NET Framework automatically releases memory for reuse by destroying objects that are no longer in use.

In C\#.NET, the garbage collector checks for the objects that are not currently in use by applications. When the garbage collector comes across an object that is marked for garbage collection, it releases the memory occupied by the object.

\section{J. Overloading}

Overloading is another feature in C\#. Overloading enables us to define multiple procedures with the same name, where each procedure has a different set of arguments. Besides using 
overloading for procedures, we can use it for constructors and properties in a class.

\section{K. Multithreading}

C\#.NET also supports multithreading. An application that supports multithreading can handle multiple tasks simultaneously, we can use multithreading to decrease the time taken by an application to respond to user interaction.

\section{Structured Exception Handling}

C\#.NET supports structured handling, which enables us to detect and remove errors at runtime. In C\#.NET, we need to use Try...Catch...Finally statements to create exception handlers. Using Try...Catch...Finally statements, we can create robust and effective exception handlers to improve the performance of our application.

\section{The .Net Framework}

The .NET Framework is a new computing platform that simplifies application development in the highly distributed environment of the Internet.

\section{Objectives of. Net framework}

1) To provide a consistent object-oriented programming environment whether object codes is stored and executed locally on Internet-distributed, or executed remotely.

2) To provide a code-execution environment to minimizes software deployment and guarantees safe execution of code.

3) Eliminates the performance problems.

4) There are different types of application, such as Windows-based applications and Web-based applications.

\section{SySTEM DESIGN}

1) Use Case Diagram

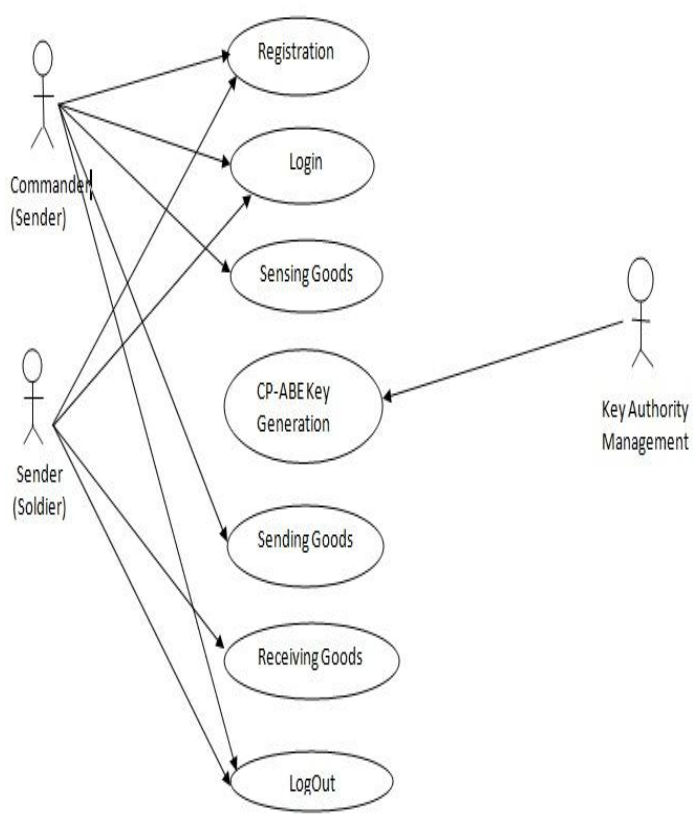

\section{2) Class Diagram}

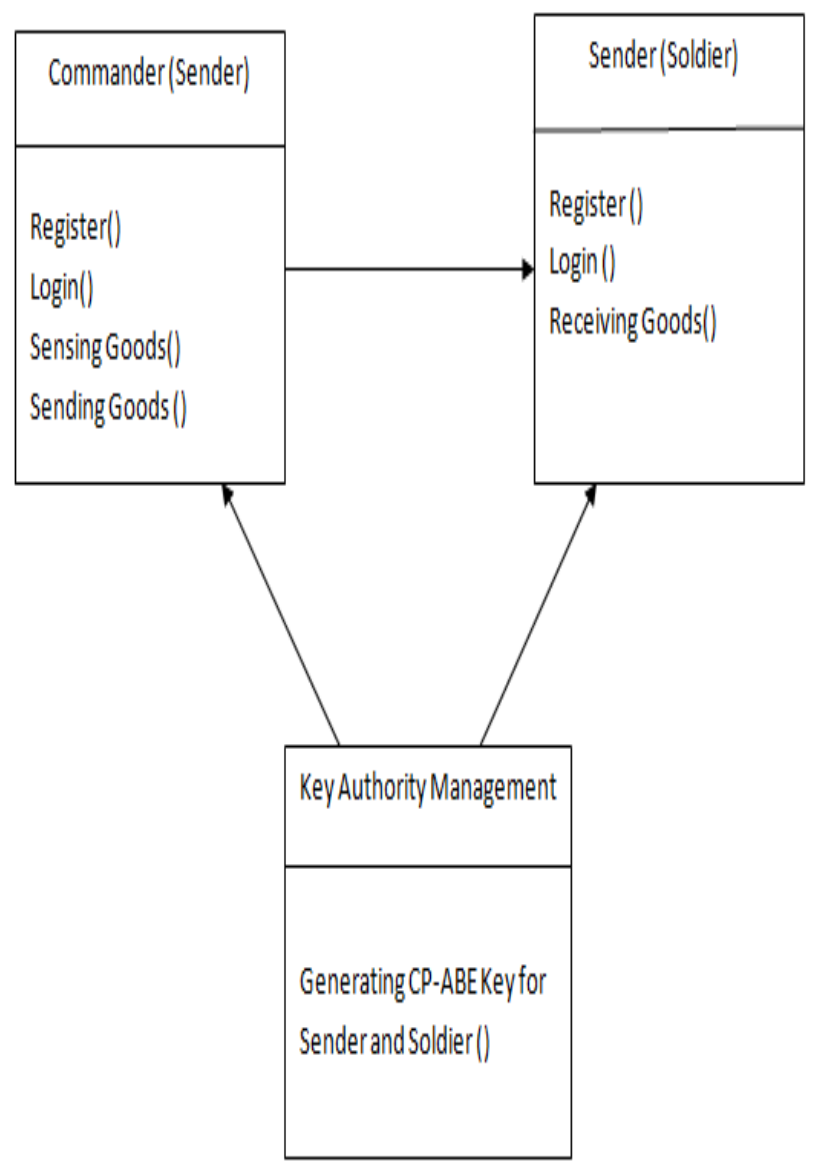

3) Data Flow Diagram

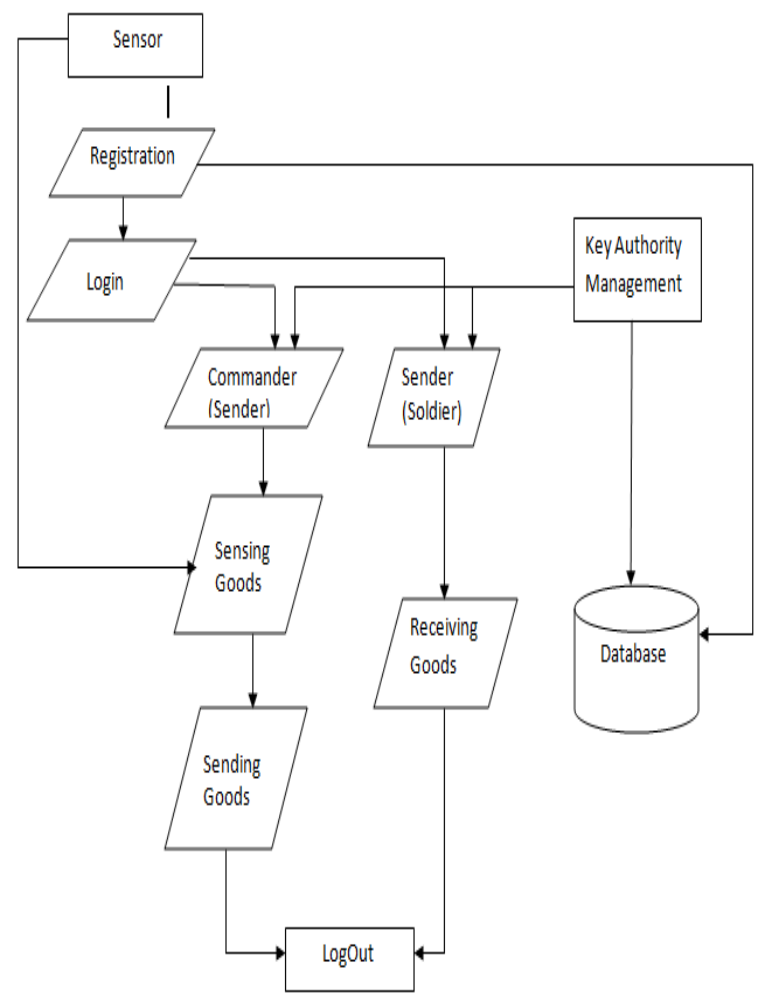




\section{4) Activity Diagram}

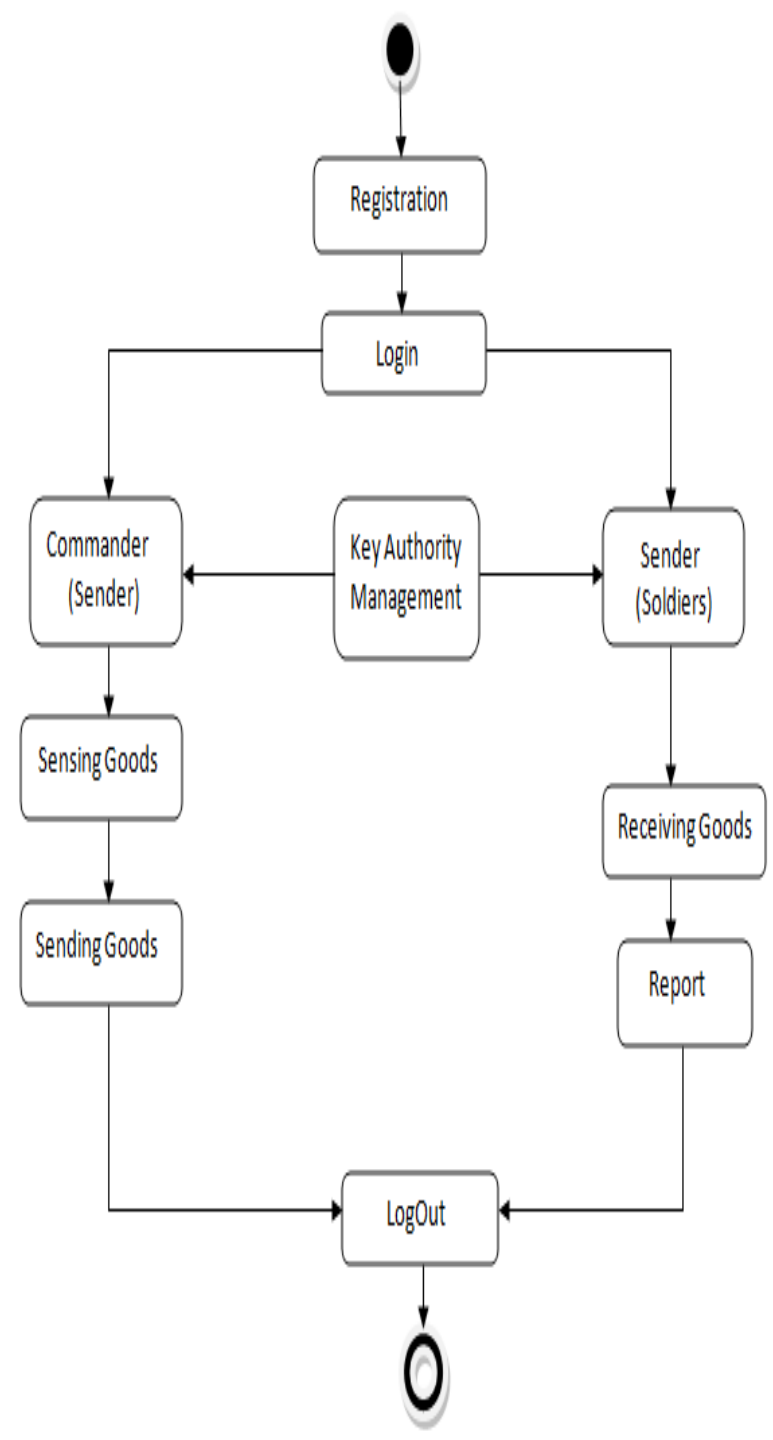

5) System Study

\section{A. Feasibility Study}

The feasibility of the project is analyzed in this phase and business proposal is put forth with a very general plan for the project and some cost estimates. During system analysis the feasibility study of the proposed system is to be carried out. This is to ensure that the proposed system is not a burden to the company. For feasibility analysis, some understanding of the major requirements for the system is essential.

Three key considerations involved in the feasibility analysis are,
A. Economical Feasibility
B. Technical Feasibility
C. Social Feasibility

A. Economical Feasibility

This study is carried out to check the economic impact that the system will have on the organization. The amount of fund that the company can pour into the research and development of the system is limited. The expenditures must be justified. Thus the developed system as well within the budget and this was achieved because most of the technologies used are freely available. Only the customized products had to be purchased.

\section{B. Technical Feasibility}

This study is carried out to check the technical feasibility, that is, the technical requirements of the system. Any system developed must not have a high demand on the available technical resources. This will lead to high demands on the available technical resources. This will lead to high demands being placed on the client. The developed system must have a modest requirement, as only minimal or null changes are required for implementing this system.

\section{Social Feasibility}

The aspect of study is to check the level of acceptance of the system by the user. This includes the process of training the user to use the system efficiently. The user must not feel threatened by the system, instead must accept it as a necessity. The level of acceptance by the users solely depends on the methods that are employed to educate the user about the system and to make him familiar with it. His level of confidence must be raised so that he is also able to make some constructive criticism, which is welcomed, as he is the final user of the system..

\section{6) System Testing}

The purpose of testing is to discover errors. Testing is the process of trying to discover every conceivable fault or weakness in a work product. It provides a way to check the functionality of components, sub assemblies, assemblies and/or a finished product It is the process of exercising software with the intent of ensuring that the

Software system meets its requirements and user expectations and does not fail in an unacceptable manner. There are various types of test. Each test type addresses a specific testing requirement.

\section{TYPES OF TESTS}

\section{A. Unit Testing}

Unit testing involves the design of test cases that validate that the internal program logic is functioning properly, and that program inputs produce valid outputs. All decision branches and internal code flow should be validated. It is the testing of individual software units of the application .it is done after the completion of an individual unit before integration. This is a structural testing, that relies on knowledge of its construction and is invasive. Unit tests perform basic tests at component level and test a specific business process, application, and/or system configuration. Unit tests ensure that each unique path of a business process performs accurately to the documented specifications and contains clearly defined inputs and expected results.

\section{B. Integration Testing}

Integration tests are designed to test integrated software components to determine if they actually run as one program. Testing is event driven and is more concerned with the basic outcome of screens or fields. Integration tests demonstrate that although the components were individually satisfaction, as 
shown by successfully unit testing, the combination of components is correct and consistent. Integration testing is specifically aimed at exposing the problems that arise from the combination of components.

\section{Functional Testing}

Functional tests provide systematic demonstrations that functions tested are available as specified by the business and technical requirements, system documentation, and user manuals.

Functional testing is centered on the following items:

Valid Input $\quad$ : $\quad$ identified classes of valid input must be accepted.

Invalid Input : $\quad$ identified classes of invalid input must be rejected.

Functions

identified functions must be exercised.

Output : identified classes of application outputs must be Systems/Procedures : interfacing systems or procedures must be invoked.

Organization and preparation of functional tests is focused on requirements, key functions, or special test cases. In addition, systematic coverage pertaining to identify Business process flows; data fields, predefined processes, and successive processes must be considered for testing. Before functional testing is complete, additional tests are identified and the effective value of current tests is determined.

\section{System Testing}

System testing ensures that the entire integrated software system meets requirements. It tests a configuration to ensure known and predictable results. An example of system testing is the configuration oriented system integration test. System testing is based on process descriptions and flows, emphasizing pre-driven process links and integration points.

\section{E. White Box Testing}

White Box Testing is a testing in which in which the software tester has knowledge of the inner workings, structure and language of the software, or at least its purpose. It is purpose. It is used to test areas that cannot be reached from a black box level.

\section{F. Black Box Testing}

Black Box Testing is testing the software without any knowledge of the inner workings, structure or language of the module being tested. Black box tests, as most other kinds of tests, must be written from a definitive source document, such as specification or requirements document, such as specification or requirements document. It is a testing in which the software under test is treated, as a black box .you cannot "see" into it. The test provides inputs and responds to outputs without considering how the software works.
G. Table Design

Commander (Sender):
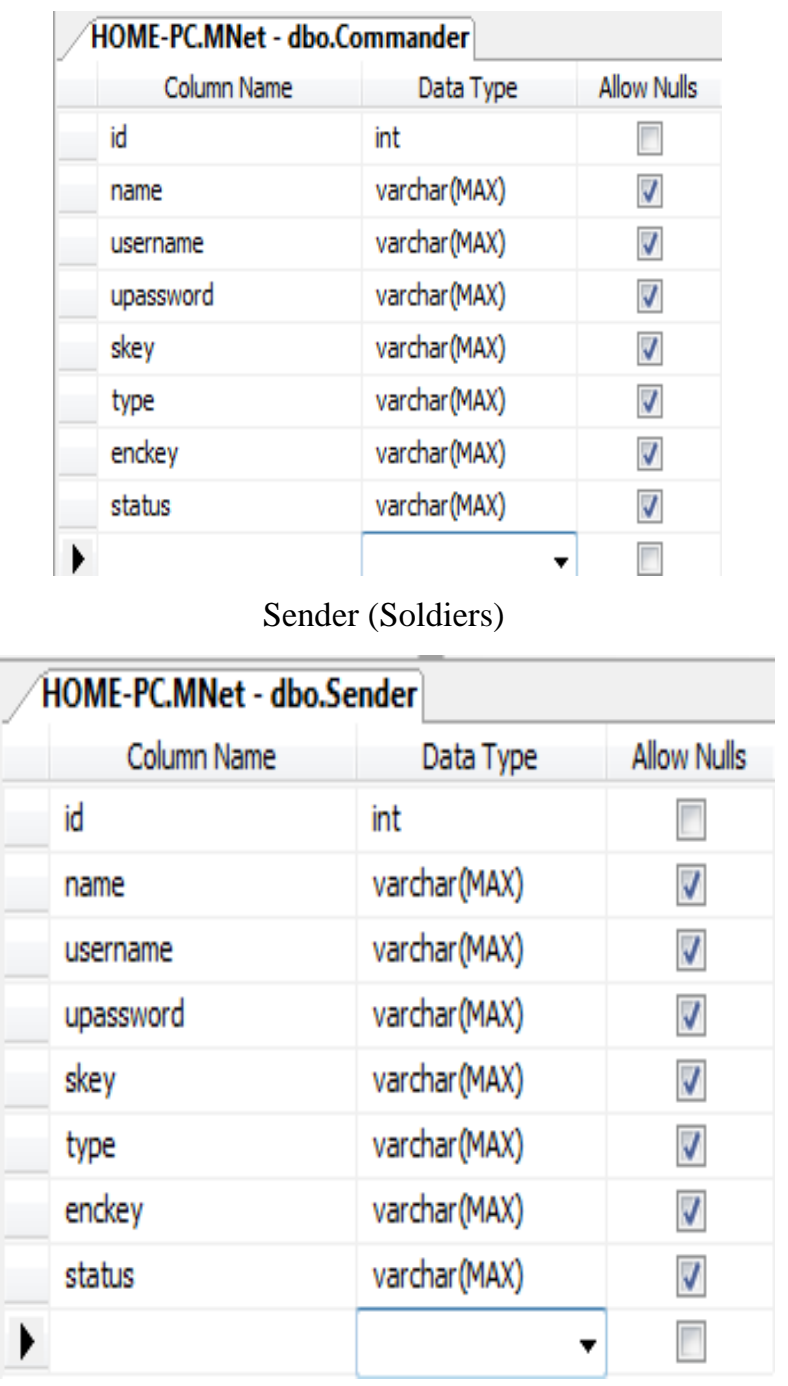

Sensors

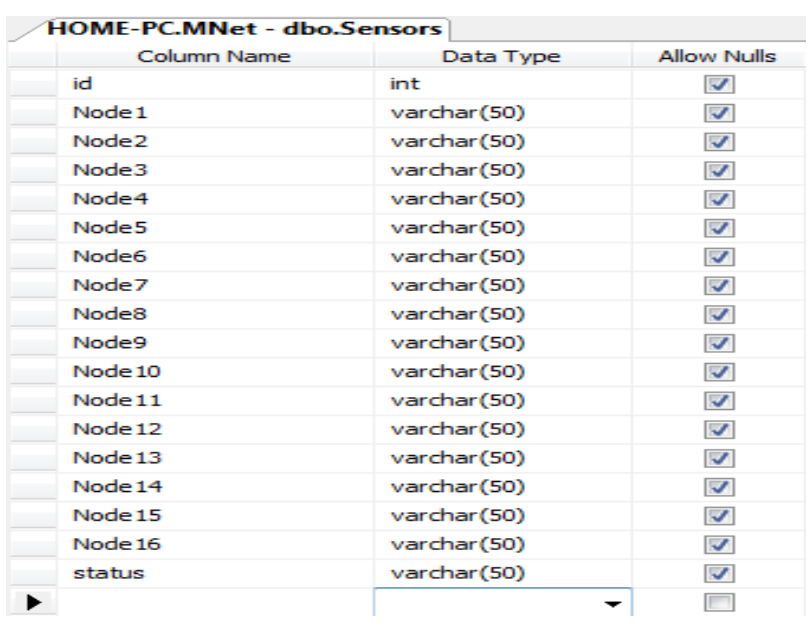

Timing 


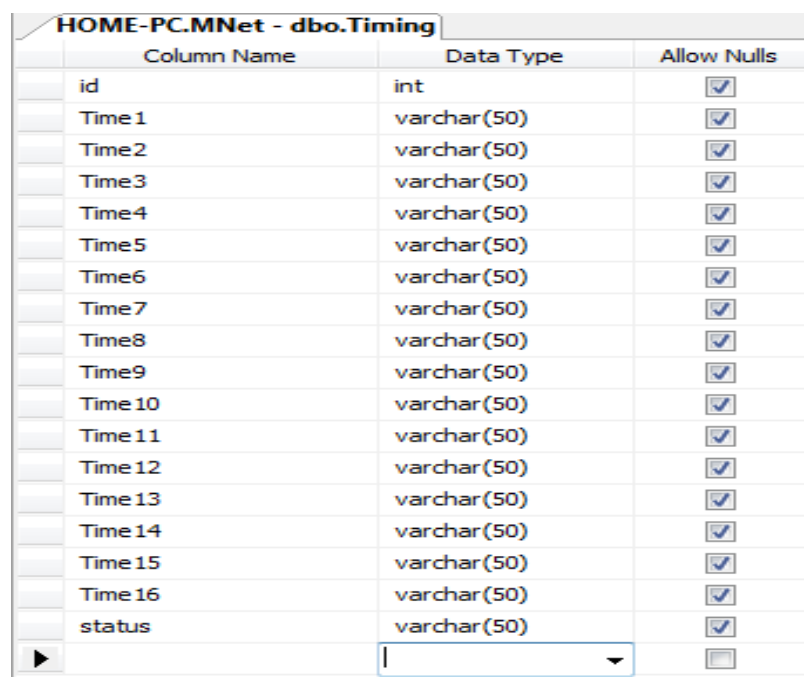

\section{CONClusion AND Future ENHANCEMENT}

DTN technologies are becoming successful solutions in military applications that allow wireless devices to communicate with each other and access the confidential information reliably by exploiting external storage nodes. $\mathrm{CP}$ $\mathrm{ABE}$ is a scalable cryptographic solution to the access control and secure data retrieval issues. In this paper, we proposed an efficient and secure data retrieval method using $\mathrm{CP}-\mathrm{ABE}$ for decentralized DTNs where multiple key authorities manage their attributes independently. The inherent key escrow problem is resolved such that the confidentiality of the stored data is guaranteed even under the hostile environment where key authorities might be compromised or not fully trusted. In addition, the fine-grained key revocation can be done for each attribute group. We demonstrate how to apply the proposed mechanism to securely and efficiently manage the confidential data distributed in the disruption- tolerant military network.

\section{REFERENCES}

[1] J. Burgess, B. Gallagher, D. Jensen and B.N. Levine, "MaxProp: Routing for Vehicle-Based Disruption-Tolerant Networks", In INFOCOM, Vol. 6, Pp. 1-11, 2006.

[2] M. Chuah and P. Yang, "Node density-based adaptive routing scheme for disruption tolerant networks", In Conference on MILCOM IEEE Military Communications, pp. 1-6, 2006.

[3] M.M. Bin Tariq, M. Ammar and E. Zegura, "Message ferry route design for sparse ad hoc networks with mobile nodes", In Proceedings of the 7th ACM international symposium on Mobile ad hoc networking and computing, pp. 37-48, 2006.

[4] S. Roy and M. Chuah, "Secure data retrieval based on ciphertext policy attribute-based encryption (CP-ABE) system for the DTNs", Lehigh CSE Tech, Vol. 8, No. S8, Pp. 542-8, 2009.

[5] M. Chuah and P. Yang, "Performance evaluation of content-based information retrieval schemes for DTNs", In Conference on MILCOM 2007-IEEE Military Communications, Pp. 1-7, 2007.

[6] M. Kallahalla, E. Riedel, R. Swaminathan, Q. Wang and K. Fu, "Plutus: Scalable Secure File Sharing on Untrusted Storage", In Fast, Vol. 3, Pp. 29-42, 2003.

[7] L. Ibraimi, M. Petkovic, S. Nikova, P. Hartel and W. Jonker, "Mediated ciphertext-policy attribute-based encryption and its application", In Information security applications, Pp. 309-323. 2009.

[8] N. Chen, M. Gerla, D. Huang and X. Hong, "Secure, selective group broadcast in vehicular networks using dynamic attribute based encryption", In Ad Hoc Networking Workshop (Med-Hoc-Net), 2010 The 9th IFIP Annual Mediterranean, Pp. 1-8, 2010.
[9] D. Huang and M. Verma, "ASPE: Attribute-based secure policy enforcement in vehicular ad hoc networks", Ad Hoc Networks, Vol. 7, No. 8, Pp. 1526-1535, 2009.

[10] Lewko, A., \& Waters, B. (2011, May). Decentralizing attribute-based encryption. In Annual International Conference on the Theory and Applications of Cryptographic Techniques (pp. 568-588). Springer Berlin Heidelberg.

[11] A. Sahai and B. Waters, "Fuzzy identity-based encryption", In Annual International Conference on the Theory and Applications of Cryptographic Techniques, Pp. 457-473, 2005.

[12] A. Sahai and B. Waters, "Fuzzy identity-based encryption", In Annual International Conference on the Theory and Applications of Cryptographic Techniques, Pp. 457-473, 2005.

[13] J. Bethencourt, A. Sahai and B. Waters, "Ciphertext-policy attribute based encryption", In 2007 IEEE symposium on security and privacy (SP'07), Pp. 321-334, 2007.

[14] R. Ostrovsky, A. Sahai and B. Waters, "Attribute-based encryption with non-monotonic access structures", In Proceedings of the 14th ACM conference on Computer and communications security, Pp. 195-203, 2007.

[15] S. Yu, C. Wang, K. Ren and W. Lou, "Attribute based data sharing with attribute revocation", In Proceedings of the 5th ACM Symposium on Information, Computer and Communications Security, Pp. 261-270, 2010

[16] A. Boldyreva, V. Goyal and V. Kumar, "Identity-based encryption with efficient revocation", In Proceedings of the 15th ACM conference on Computer and communications security, Pp. 417-426, 2008.

[17] M. Pirretti, P. Traynor, P. McDaniel and B. Waters, "Secure attributebased systems", Journal of Computer Security, Vol. 18, No. 5, Pp. 799837, 2010.

[18] S. Rafaeli and D. Hutchison, "A survey of key management for secure group communication", ACM Computing Surveys (CSUR), Vol. 35, No. 3, Pp. 309-329, 2003.

[19] S. Mittra, "Iolus: A framework for scalable secure multicasting", In ACM SIGCOMM Computer Communication Review, Vol. 27, No. 4, Pp. 277-288, 1997.

[20] J. Staddon, P. Golle, M. Gagné and P. Rasmussen, “A content-driven access control system", In Proceedings of the 7th symposium on Identity and trust on the Internet, Pp. 26-35, 2008.

[21] L. Cheung and C. Newport, "Provably secure ciphertext policy ABE", In Proceedings of the 14th ACM conference on Computer and communications security, Pp. 456-465, 2007.

[22] V. Goyal, A. Jain, O. Pandey and A. Sahai, "Bounded ciphertext policy attribute based encryption", In International Colloquium on Automata, Languages, and Programming, Pp. 579-591, 2008.

[23] X. Liang, Z. Cao, H. Lin and D. Xing, "Provably secure and efficient bounded ciphertext policy attribute based encryption", In Proceedings of the 4th International Symposium on Information, Computer, and Communications Security, pp. 343-352, 2009.

[24] M, Chase and S.S. Chow, "Improving privacy and security in multiauthority attribute-based encryption", In Proceedings of the 16th ACM conference on Computer and communications security, Pp. 121-130, 2009.

[25] M. Chase, "Multi-authority attribute based encryption", In Conference of Theory of Cryptography, Pp. 515-534, 2007. 